\title{
The Help of HPV Integration Testing to Avoid the Misdiagnosis of a Patient with Stage lal Cervical Cancer: A Case Report and Literature Review
}

Jialu $\mathrm{Li}^{\prime}$

Xiaoli Zhang ${ }^{2}$

Pengpeng Wang ${ }^{3}$

Weiping $\mathrm{Li}^{4}$

'Department of Obstetrics and Gynecology, The Second School of Clinical Medicine, Southern Medical University, Hainan Hospital of PLA General Hospital, Hainan, People's Republic of China; ${ }^{2}$ Department of Obstetrics and Gynecology, Three Medical Center, Chinese PLA General Hospital, Beijing, People's Republic of China; ${ }^{3}$ MyGenostics Inc., Beijing, People's Republic of China; ${ }^{4}$ Department of Obstetrics and Gynecology, Seven Medical Center, Chinese PLA General Hospital, Beijing, People's Republic of China
Correspondence: Weiping Li

Department of Obstetrics and

Gynecology, Seven Medical Center,

Chinese PLA General Hospital, Beijing,

People's Republic of China

Email liweiping258@sina.com

\begin{abstract}
Screening and prevention in the early stage of cervical cancer could improve the 5 -year survival rate of cervical cancer by up to $90 \%$. The occurrence rate of human papillomavirus (HPV) integration has gradually increased with the development of cervical lesions. Here, we report the case of a 43-year-old woman diagnosed with chronic cervicitis based on cervical biopsy results. After medical consultation, surgery was recommended to the patient considering her $64 \mathrm{HPV}$ integration sites and other biochemical and clinical test results. The pathology result of the conical biopsy obtained during operation confirmed that both her cervix and the glands had high-grade squamous intraepithelial lesion or cervical intraepithelial neoplasia III (HSIL/CIN III). The patient underwent uterectomy and bilateral salpingectomy and was discharged thereafter. The diagnosis of the patient was revised to stage Ial cervical cancer. The number of HPV integration sites is suggested to be an auxiliary indicator in the early screening of cervical cancer for predicting high-grade CIN and invasive cervical cancer.
\end{abstract}

Keywords: HPV DNA, DNA methylation detection, cervical cancer, precancerous lesion of cervical cancer

\section{Introduction}

Cervical cancer is one of the most common gynecological malignant tumors that seriously threatens women's health. In recent years, the incidence and mortality of cervical cancer have increased, while the age of onset is decreasing in China. ${ }^{1}$ Therefore, accurate screening and prevention in the early stages of cervical cancer in the context of precision medicine is necessary. Considering that chronic persistent infection with the high-risk human papillomavirus (HPV) is one of the main causes of cervical cancer, the detection of high-risk HPV DNA accompanied by liquid-based cytology (ThinPrep cytologic test [TCT]) and colposcopy examination are the main screening methods for cervical lesions in clinical settings. Although continuous infection with HPV is almost a necessary condition for cervical cancer, not all infected people develop cervical cancer. ${ }^{2}$ An indicator for cervical lesions is urgently needed to help identify high-risk groups in clinical settings. Ho et al confirmed that the frequency of integration between the high-risk HPV DNA and the host genome was increased following cervical lesions. ${ }^{3}$ Melsheimer et al have confirmed that the integration event could lead to instability of the host genome and abnormal gene expression of both viral and host cells. ${ }^{4,5}$ Thus, the number of HPV 
integration sites might be a potential indicator of malignant progression from cervical intraepithelial neoplasia to cervical cancer. ${ }^{6}$ In this study, we report the case of a 43year-old woman who was diagnosed with chronic cervicitis based on cervical biopsy results at onset, but was finally diagnosed with stage Ia1 cervical cancer after considering the number of HPV integration sites as an indicator and underwent proper treatment.

\section{Case Presentation}

\section{Ethics Approval and Consent to Participate}

The Medical Ethics Committee of Southern Medical University approved the study (ethical approval number: HN20200108). The patient has signed an informed consent to submit her tumor samples and paired peripheral blood for gene testing and scientific research and to publish her case details.

Here, we present a 43-year-old woman who was admitted to the Hainan Hospital of PLA General Hospital with a two-month history of HPV 16 infection. The patient had a small amount of thin yellow secretion and no history of postcoital bleeding. First, she underwent a gynecological examination, which showed a smooth cervix without obvious abnormalities. Second, she underwent TCT and found no significant atypical cell changes. She underwent cervical biopsy according to the diagnostic process. The diagnosis after cervical biopsy was chronic cervicitis (The cervix is 3, 6, 9 and 12 o 'clock clockwise). In order to rule out the possibility of malignant lesions, the patient underwent HPV typing and integration tests, the P16 test, and PAX1 methylation detection. The patient accepted relieve from anxiety. She had HPV 16 infection with 64 HPV DNA integration sites. The P16 test was positive, and the value of PAX1 methylation was 1.01, indicating a high risk for cervical cancer. The patient underwent surgery after medical counseling. The surgery was performed based on both the results of the tests and the desire of the patient.

After performing conization of the uterine cervix, the possibility of endogenous lesions combined with the result of the hysteroscopy was excluded. The pathology result of the conical biopsy obtained during operation indicated that both her cervix (from 1 to 12 points) and the glands had high-grade squamous intraepithelial lesion or cervical intraepithelial neoplasia III (HSIL/CIN III). 1 o 'clock clockwise of the cervix presented with budding manifestations, indicating microinvasive carcinoma. There were some retained cysts with both depth and width of less than $0.01 \mathrm{~cm}$. CIN was found at the incisor edges at 1 point and 6-8 points. The patient underwent further examination for tumor markers after surgery. The values of Ca19-9 (normal reference value: $0.1-37 \mu / \mathrm{mL}$ ) and CA724 (normal reference value:0.1-30 $\mu / \mathrm{mL}$ ) were $39.79 / \mathrm{mL}$ and $37.290 / \mathrm{mL}$, respectively. The normal reference values were provided by the Department of Clinical Laboratory of the Hainan Hospital of PLA General Hospital. Pelvic computed tomography (CT) showed a change in the uterine cervix, which indicated the manifestations of cervical cancer. Considering her medical history and auxiliary examination, both uterectomy and bilateral salpingectomy were performed, and the patient was finally diagnosed with stage Ia1 cervical cancer. Postoperative pathology examination revealed a small amount of HSIL/CINIII residue at three different points in her cervix and gland, and no infiltration was observed. According to the immunohistochemistry results, the Ki$67 \times 2$ of the basement membrane was positive. No cancer cells were found in the parametrium or incisional margins of the vaginal wall. The operation was successful, and the patient was discharged.

\section{Discussion}

In this case, the patient was possibly followed up based on her cervical biopsy result only, and there was no clear indication for surgery according to the guidelines. In the absence of intervention, early cervical cancer can progress to more severe cervical cancer as the disease progresses. With a good follow-up, the patient may be diagnosed early with cervical cancer, but in the case of poor follow-up, the diagnosis may be delayed and may progress to more advanced cervical cancer. However, her 64 HPV integration sites combined with other molecular detection results indicated a high risk for cervical cancer, which was subsequently confirmed by her postoperative pathology test. This indicates that the number of HPV integration sites could be a potential indicator for cervical cancer and could be used for clinical diagnosis and to guide treatment. This can help to reduce the number of missed diagnoses.

Studies have reported that the coincidence rate of cervical biopsy and postoperative pathological biopsy is approximately $90 \%{ }^{7,8}$ The location and extent of the sampling, as well as the age of the patient, may result in false negatives in cervical biopsy. ${ }^{10}$ In this case, the patient underwent cervical biopsy twice before surgery, and the 
results were negative. Studies have reported that the overall sensitivity, specificity, positive predictive value, negative predictive value, and accuracy of cervical exfoliation cytological smear for CIN2+ were 81.0\%, 95.4\%, 38.3\%, $99.3 \%$, and $94.9 \%$, respectively, based on the cervical biopsy results of 25,830 women with abnormal cervical cells and HPV positivity. ${ }^{9}$ Studies have reported that secondary colposcopy could significantly improve the detection rate of CIN2. ${ }^{1}$ The gynecologists might make mistakes during operation, although studies have reported that the level of training of gynecologists did not have any substantial effect on the accuracy. ${ }^{10}$

Additionally, considering that our patient was 45 years old, the presence of a cervical squamous columnar epithelium transformation zone might have led to the falsenegative results. Studies have reported that the sensitivity of colposcopy for the diagnosis of microinvasive carcinoma in the cervix was only approximately $23 \%$, and $14.5 \%$ of microinvasive cancers were hidden in the type III transformation zone. ${ }^{11}$ Considering that the type III transformation zone is located in the cervical canal, colposcopy is inadequate. Cervical tube scratching and multipoint cervical biopsy cannot provide sufficient reliable information, which might lead to a misdiagnosis, especially when the patient is infected with HPV 16 or $18 .^{12}$ Thus, it was necessary to combine cytology, cervical biopsy, HPV testing, and other potential biomarkers to make a final diagnosis.

In this case, we consider the number of viral integration sites as a biomarker to evaluate the risk of cancer in the host. It is known that the integration between viral DNA and the host genome is important for the occurrence of cervical cancer. The greater the number of integration sites, the more affected the host genome. Tsakogiannis et al confirmed that only the integration of HPV-DNA with the chromosomes in cervical epithelial cells can cause host cell gene mutation, leading to cervical cancer. ${ }^{14}$ Sun et al confirmed that the virus detection rates of high-risk HPV, including HPV16 and HPV18 in cervical cancer, HSIL, and LSIL were $88.2 \%, 76.2 \%$, and $37 \%$, respectively. ${ }^{15}$ Huang et al showed that HPV integration breakpoints occurred in $97.8 \%$ of the CC samples, $70.5 \%$ of the CIN samples, and $42.8 \%$ of the normal cervical samples with HPV infection. ${ }^{19}$ Concerning the carcinogenic mechanism of HPV-DNA integration, studies have shown that, from the perspective of gene structure changes, the rupture and integration of the E1 and E2 regions of the open reading frame of HPV can lead to damage or deletion of the E1 and E2 genes and abnormal expression of the E1 and E2 proteins. The transcriptional inhibitory effect of E2 on E6 and E7 was decreased, and E6 and E7 were expressed in large numbers and combined with the tumor suppressor genes p53 and pRb to form a complex, resulting in the loss of tumor suppressor effect, abnormal cell cycle regulation, cell stagnation in G1 phase, infinite proliferation, cell immortality, and finally canceration. ${ }^{16}$ At the chromosomal level, viral gene integration damages the host cell chromosomes and destabilizes them. This leads to the inactivation of oncogenic and tumor suppressor groups. After HPV-DNA integrates into the host cell chromophores, oncogenes such as MYC and HMGA2 are overexpressed, and the expression of tumor suppressor genes such as FHIT and LRP1B is downregulated. The cells in the upper uterine cervix are transformed and carcinogenesis is induced. ${ }^{17,18}$

Studies have revealed other potential indicators of HPV DNA integration for cancer risk evaluation, such as the integration of hot integration and viral/host genes. For example, $\mathrm{Hu}$ et al reported new hot spots (shMGA2 [7.8\%], DLG2 [4.9\%], and Sema3D [4.9\%]) and potential mechanisms by drawing a genome-wide, unbiased, singlenucleotide resolution integration map of HPV in CIN and cervical cancer. Huang et al reported eight genes with significant mutations in cervical cancer by whole genome sequencing or whole exome sequencing on 102 tumornormal pairs, and four of those genes (FAT1, MLL3, MLL2, and FADD) had not been previously reported. In addition, some studies have reported that the viral load of high-risk HPV is positively correlated with the severity of cervical lesions. ${ }^{13}$ However, the cutoff value of viral load for risk evolution or diagnosis is unclear, and further studies are required. In fact, the number of HPV DNA integration sites as an indicator focused on the same problem. Many studies are required in the future.

With the application of new cancer intervention concepts and technologies, the HPV gene integration test will help clinicians to predict the outcome of patients infected with the HPV at an early stage, intervene before the development of cancer, and achieve the purpose of early detection and treatment of cervical cancer. With the help of the gene test, missed diagnosis and panic in patients could be reduced, and patient satisfaction could improve. As a supplementary indicator of early cervical cancer screening, the number of HPV DNA integration sites can help medical doctors in the diagnosis and treatment. More medical resources should be focused 
on high-risk patients. Both psychological pressure and economic burden of patients brought about by cervical cancer screening and HPV vaccination could be reduced. Therefore, this detection method has great clinical significance. In addition, more attention should be paid to patients with normal biopsy but more gene integration sites, especially women in menopause and to alert concerning the occurrence of early cervical cancer. Thus, further research on the relationship between cervical lesions and the number of integration sites is required in the future. At present, most of the reported integration sites occur in cervical cancer, and the study on integration sites in early cervical cancer lesions is still in the exploratory stage. Large cohorts of multicenter clinical studies should be conducted in future studies. Exploring new integration sites will lay a solid foundation for finding the laws behind the integration of HPV genes.

\section{Conclusion}

The patient benefited from the detection of HPV integration. The number of HPV integration sites is suggested to be an auxiliary indicator in the early screening of cervical cancer for predicting high-grade CIN and invasive cervical cancer. However, there are still many unexplored areas for HPV integration. With the continuous improvement of detection methods, it will guide future clinical work and play more roles in the future.

\section{Abbreviations}

HSIL/CIN III, high-grade squamous intraepithelial lesion/ cervical intraepithelial neoplasia III; HPV, human papillomavirus; TCT, ThinPrep cytologic test; CT, computed tomography; DNA, deoxyribonucleic acid.

\section{Ethics Approval and Consent to Participate}

The study was approved by the Ethics Committee of the Hainan Hospital of PLA General Hospital and was performed in accordance with the Declaration of Helsinki. All participants agreed to participate and signed informed consent.

\section{Acknowledgments}

The authors thank all the participants in this study. We are also grateful to the hospital staff who contributed to the sample and data collection.

\section{Author Contributions}

All authors made a significant contribution to the work reported, whether that is in the conception, study design, execution, acquisition of data, analysis and interpretation, or in all these areas; took part in drafting, revising or critically reviewing the article; gave final approval of the version to be published; have agreed on the journal to which the article has been submitted; and agree to be accountable for all aspects of the work.

\section{Funding}

This project was approved and funded by health Bureau of Logistics Support Department, Military Commission, General Hospital of PLA, and applied basic research Project (Grant No.:19JSZ21).

\section{Disclosure}

The authors have declared that no competing interest exists.

\section{References}

1. Valdez M, Jeronimo J, Bansil P, et al. Effectiveness of novel, lower cost molecular human papillomavirus-based tests for cervical cancer screening in rural China. Int $J$ Cancer. 2016;138(6):1453-1461. doi:10.1002/ijc. 29877

2. Bray F, Ferlay J, Soerjomataram I, Siegel RL, Torre LA, Jemal A. Global cancer statistics 2018: GLOBOCAN estimates of incidence and mortality worldwide for 36 cancers in 185 countries. CA Cancer J Clin. 2018;68(6):394-424. doi:10.3322/caac.21492

3. Ho CM, Lee BH, Chang SF, et al. Integration of human papillomavirus correlates with high level of viral oncogene transcripts in cervical carcinogenesis. Virus Res. 2011;161(2):124-130. doi:10.1016/j. virusres.2011.06.012

4. Melsheimer P, Vinkokurova S, Wentzensen N, Bastert G, von Knebel Doeberitz M. DNA aneuploidy and integration of human papillomavirus type $16 \mathrm{e} 6 / \mathrm{e} 7$ oncogenes in intraepithelial neoplasia and invasive squamous cell carcinoma of the cervix uteri. Clin Cancer Res. 2004;10 (9):3059-3063. doi:10.1158/1078-0432.CCR-03-0565

5. Vinokurova S, Wentzensen N, Kraus I, et al. Type-dependent integration frequency of human papilloma virus genomes in cervical lesions. Cancer Res. 2008;68(1):307-313. doi:10.1158/0008-5472.CAN-072754

6. Origoni M, Cristoforoni P, Carminati G, et al. E6/E7mRNA testing for human papilloma virus-induced highgrade cervical intraepithelial disease (CIN2/CIN3): a promising perspective. E Cane Med Sci. 2015;9:533.

7. Fu ZC, Wang FM, Cai JM. Gene expression changes in residual advanced cervical cancer after radiotherapy: indicators of poor prognosis and radioresistance. Med Sci Monit. 2015;21(2):1276-1287. doi:10.12659/MSM.893689

8. Chacko S. Effect of structured teaching programme on VIA test for early detection and diagnosis of cervical cancer. Nurs J India. 2014; 105(5):221-224.

9. Pan QJ, Hu SY, Zhang X, et al. Pooled analysis of the performance of liquid-based cytology in population-based cervical cancer screening studies in China. Cancer Cytopathol. 2013;121(9):473-482. doi:10.10 02/cncy. 21297 
10. Stuebs FA, Schulmeyer CE, Mehlhorn G, et al. Accuracy of colposcopy-directed biopsy in detecting early cervical neoplasia: a retrospective study. Arch Gynecol Obstet. 2019;299(2):525-532. doi:10.1007/s00404-018-4953-8

11. Furtado Y, Almeida G, Lima R, et al. Microinvasive squamous carcinoma (FIGO stage IA1) of the cervix: are there colposcopic criteria for the diagnosis? Am J Obstet Gynecol. 2011;205(4):360. e1-360.e4. doi:10.1016/j.ajog.2011.06.036

12. Josefsson AM, Magnusson PK, Ylitalo N, et al. Viral load of human papilloma virus 16 as a determinant for development of cervical carcinoma in situ: a nested case-control study. Lancet. 2000;355 (9222):2189-2193. doi:10.1016/S0140-6736(00)02401-6

13. Shen G, Cheng J, Wang Y, Zhou P, Viral ZG. DNA load of high-risk human papilloma virus is closely associated with the grade of cervical lesions. Int J Clin Exp Med. 2014;7(12):5826-5831.

14. Tsakogiannis D, Kyriakopoulou Z, Ruether IG, et al. Determination of human papillomavirus 16 physical status through E1/E6 and E2/E6 ratio analysis. $J$ Med Microbiol. 2014;63(Pt12):1716-1723. doi:10. 1099/jmm.0.076810-0
15. Sun C, Reimers LL, Burk RD. Methylation of HPV16 genome CpG sites is associated with cervix precancer and cancer. Gynecol Oncol. 2011;121(1):59-63. doi:10.1016/j.ygyno.2011.01.013

16. Moody CA, Laimins LA. Human papilloma virus on coproteins: pathways to transformation. Nat Rev Cancer. 2010;10(8):550-560. doi:10.1038/nrc2886

17. Schmitz M, Driesch C, Beer-Grondke K, et al. Loss of gene function as a consequence of human papilloma virus DNA integration. Int J Cancer. 2012;131(5):E593-E602. doi:10.1002/ijc.27433

18. Pett M, Coleman N. Integration of high-risk human papillomavirus: a key event in cervical carcinogenesis. J Pathol. 2007;212 (4):356-367. doi:10.1002/path.2192

19. Huang J, Qian Z, Gong Y, et al. Comprehensive genomic variation profiling of cervical intraepithelial neoplasia and cervical cancer identifies potential targets for cervical cancer early warning. $J \mathrm{Med}$ Genet. 2019;56(3):186-194. doi:10.1136/jmedgenet-2018-105745

\section{Publish your work in this journal}

Pharmacogenomics and Personalized Medicine is an international, peer-reviewed, open access journal characterizing the influence of genotype on pharmacology leading to the development of personalized treatment programs and individualized drug selection for improved safety, efficacy and sustainability. This journal is indexed on the American Chemical Society's Chemical Abstracts Service (CAS). The manuscript management system is completely online and includes a very quick and fair peer-review system, which is all easy to use. Visit http://www.dovepress.com/testimonials.php to read real quotes from published authors. 